\title{
Improving simultaneously the quality and safety of cooked and peeled shrimp using a cocktail of bioprotective lactic acid bacteria
}

\author{
Saraoui Taous ${ }^{1,2,3}$, Cornet Josiane ${ }^{1}$, Guillouet Emilie ${ }^{1}$, Pilet Marie-France ${ }^{2,3}$, Chevalier Frédérique ${ }^{1}$, \\ Joffraud Jean-Jacques ${ }^{1}$, Leroi Françoise ${ }^{1,{ }^{*}}$
}

${ }^{1}$ Laboratoire Ecosystèmes Microbiens et Molécules Marines pour les Biotechnologies (EM3B), Ifremer, Rue de l'lle d'Yeu, 44311 Nantes Cedex 03, France

${ }_{2}^{2}$ LUNAM Université, Oniris, UMR 1014 Secalim, Site de la Chantrerie, F-44307 Nantes, France

${ }^{3}$ INRA, F-44307 Nantes, France

* Corresponding author : Françoise Leroi, email address : Francoise.Leroi@ifremer.fr

taous.saraoui@gmail.com ; josiane.cornet@ifremer.fr ; emilieguillouet@hotmail.fr ; marie-france.pilet@oniris-nantes.fr ; fchevali@ifremer.fr ; jean.jacques.joffraud@ifremer.fr

\begin{abstract}
:
Tropical shrimp is of considerable economic importance in the world but is highly perishable due to microbial and chemical degradation. Biopreservation is a food preservation technology based on the addition of "positive" bacteria able to kill or prevent the growth of undesirable microorganisms. Two strains of lactic acid bacteria (LAB) have previously been selected for a biopreservation strategy: Lactococcus piscium CNCM I-4031, for its ability to prevent the sensory deterioration of seafood and Carnobacterium divergens V41, which inhibits growth of Listeria monocytogenes. The objective was to test the association of the two strains to improve both the quality and safety of shrimp. In a first trial, the two LAB were inoculated alone or in a cocktail in cooked and peeled shrimp (CPS) Penaeus vannamei at $5 \times 10^{5} \mathrm{CFU} / \mathrm{g}$. Chemical, sensory and microbiological analyses by culture-dependent and independent methods were performed during storage under modified atmosphere packaging (MAP) at $8{ }^{\circ} \mathrm{C}$. The results were compared to a non-inoculated batch. In a second trial, the same experiments were repeated in the presence of $10^{2} \mathrm{CFU} / \mathrm{g}$ of $L$. monocytogenes RF191. The microbiota of CPS was composed of LAB, Shewanella spp. and Enterobacteriaceae. Brochothrix thermosphacta was not detected. L. piscium and C. divergens reached $10^{8}$ and $10^{9} \mathrm{CFU} / \mathrm{g}$, respectively, in 7 days and did not inhibit each other when co-inoculated. L. piscium reduced L. monocytogenes by 1 Log (CFU/g) for 28 days. C. divergens had an immediate listericidal effect lasting 7 days. A regrowth of $L$. monocytogenes was then observed but the count was always 2 to 5 Log (CFU/g) lower than in the control. No additional or synergic effect between protective strains was observed and the cocktail had the same inhibitory effect as $C$. divergens alone. $C$. divergens was very effective at preventing the sensory deterioration of CPS. This may be related to the inhibition of Shewanella and Enterobacteriaceae. However, the panelists could detect the presence of $C$.divergens during the first 10 days of storage, with slight unpleasant odors and flavors. L. piscium improved the sensory quality of CPS for 14 days only. In co-culture, L. piscium eliminated the off-odors and flavors released by $C$.
\end{abstract}


divergens in the early stage of storage and the co-culture allowed maintaining a good quality of CPS throughout the storage. Therefore, the use of a cocktail of L. piscium CNCM I-4031 and C. divergens V41 is recommended in a strategy of biopreservation of shrimp.

\section{Highlights}

A cocktail of $L$. piscium and $C$. divergens was tested to biopreserve cooked shrimp. $\longrightarrow$. piscium CNCM-14031 and $C$. divergens V41 have no antagonist effect on each other. $L$. piscium inhibited $L$. monocytogenes by $1 \mathrm{Log}$ and $C$. divergens by 2 to $5 \mathrm{Log}$ CFU/g. The cocktail showed no additional or synergetic effect on inhibition of Listeria. The cocktail was more efficient to improve sensory quality than the strains alone.

Keywords : Carnobacterium divergens V41, Lactococcus piscium CNCM I-4031, Penaeus vannamei, Listeria monocytogenes, Biopreservation 


\section{Introduction}

Shrimp has long been considered a rare, refined and expensive food. With the development of aquaculture, the shrimp market has increased rapidly (FAO, 2012). In 2014, the EU imported 557,700 t of shrimp between January and September (FAO-Globefish, 2015). The main suppliers of raw shrimp to Europe are Ecuador, Thailand and Greenland. Most of the imported tropical raw shrimp is processed in Europe to be sold as ready-to-eat (RTE) products, such as cooked or peeled/cooked shrimp with or without spices, and is packed under vacuum or modified atmosphere. These lightly-preserved shrimp are highly perishable due to microbiological and biochemical degradation (Dalgaard and Jørgensen, 2000). The microbial ecosystem of tropical shrimp is composed of Gram-positive bacteria (Carnobacterium sp., Enterococcus sp., Vagococcus sp., B. thermosphacta) and Gram-negative bacteria such as Enterobacteriaceae (Serratia liquefaciens) (Jaffrès et al., 2011, 2009; Laursen et al., 2006; Macé et al., 2014; Mejlholm et al., 2005). Some of them, called specific spoilage organisms, are responsible for unpleasant odors and flavors making the product inconsumable. Besides the associated economic losses, the presence of human pathogenic bacteria, such as $L$. monocytogenes, is a public health concern. $L$. monocytogenes is responsible for foodborne listeriosis, a rare but severe disease generally associated with a high mortality rate $(20-30 \%)$. It infects all human population groups but those at greatest risk are pregnant women, newborn infants, elderly people and immune-compromised patients (diabetics, cancer sufferers, etc.) (Lecuit et al., 2015). Despite the development of the HACCP system, the number of cases has increased in the last decade from 3.1 cases/million people in 2001 to 5.3 in 2014 (Lecuit et al., 2015). This bacterium constitutes a major problem in refrigerated RTE marine food (Jami et al., 2014) due to its ability to grow in a wide range of temperatures $\left(-1.5\right.$ to $\left.+45^{\circ} \mathrm{C}\right)$, salt concentrations (up to $\left.12 \%\right)$, water activity (>0.92) and pH values (4.5 - 9.0) (Gandhi and Chikindas, 2007). The prevalence and growth of $L$. monocytogenes in shrimp have been documented (Matamoros et al., 2009a; Mejlholm et al., 2005; 
Rutherford et al., 2007; Zarei et al., 2011) leading to the conclusion that, if present, L. monocytogenes can exceed the legal safety limit in EU Member States (100 CFU/g until the end of shelf-life).

Additives such as benzoic acid (E210) and sorbic acid (E200) or their derived salts, sodium benzoate (E211) and sodium sorbate (E201), are mostly used to prevent bacterial growth on shrimp. However, these products have an influence on taste and might also have negative consequences for human health due to e.g. possible pseudo-allergic reactions (Ortolani et al., 1999). Biopreservation is an alternative technology that involves natural and selected protective microorganisms to eliminate or reduce the growth of undesirable microorganisms. Many studies have reported promising results for seafood products (for reviews, see Calo-Mata et al., 2007 ; Ghanbari et al., 2013 ; Pilet and Leroi, 2011). Some protective cultures with a specific action on spoilage bacteria or pathogens have been commercialized (LLO by Biocéane and Lyoflora FP18 by Sacco S.r.I) and are currently used in cold-smoked salmon (CSS) and MAP shrimp. Currently, there is no European regulation on protective cultures in unfermented ready-to-eat food. In 2007, the European Food Safety Authority (EFSA) adopted guidelines for Qualified Presumption of Safety (QPS) that can be referred to as the European equivalent of the American GRAS status in terms of risk assessment (EFSA, 2007). In our laboratory (EM3B/Ifremer and Secalim/Oniris), one strain of $C$. divergens V41 isolated from trout viscera by Pilet et al. (1995) has been extensively studied for its anti-listeria activity. In sterile CSS, C. divergens V41, inoculated at $10^{5} \mathrm{CFU} / \mathrm{g}$, maintained $L$. monocytogenes at less than 50 (CFU/g) for 4 weeks of vacuum-storage at $4-8^{\circ} \mathrm{C}$ (Brillet et al., 2005). This inhibitory effect was due to the production of a class lla bacteriocin, divercine V41 (Métivier et al., 1998). A second LAB, L. piscium CNCM I-4031 also referenced as EU2241, has been selected for its capacity to improve the sensory quality of CSS under VP and CPS under MAP (Fall et al., 2012; Matamoros et al., 2009a). This may be due to the inhibition of $B$. thermosphacta, a spoilage bacteria of refrigerated meat and seafood, which has been detected in artificially contaminated CPS and CSS (Fall et al., 2010a; Leroi et al., 2015). Therefore, a cocktail of $C$. divergens V41 and L. piscium CNCM I-4031 seems a promising 
solution to prevent simultaneously the sensory deterioration and growth of L. monocytogenes. However, the two strains must not inhibit each other and not develop off-odors when co-cultured. In fact, two bacteria that do not spoil CSS when cultivated alone were shown to release strong unpleasant odors when tested in combination, probably due to metabiosis phenomena (Joffraud et al., 2006). In this study, challenge tests were performed to evaluate the effect of a cocktail of $C$. divergens V41 and L. piscium CNCM I-4031 on the quality and safety of CPS stored under MAP at $8^{\circ} \mathrm{C}$ for 28 days. The results were compared to a control (not inoculated with protective $L A B$ ) and to CPS inoculated with $C$. divergens and L. piscium alone. The effect of this cocktail on the quality of shrimp and on the endogenous microorganisms was evaluated by sensory evaluation of off-odors, flavors and texture and by cultural and non-cultural microbial analysis. The role of the protective LAB in L. monocytogenes was studied on CPS (with its natural endogenous ecosystem) artificially inoculated with the pathogen.

\section{Materials and methods}

\subsection{Bacterial strains}

The bioprotective strains C. divergens V41 and L. piscium CNCM I-4031 were isolated from trout and from fresh MAP salmon steak, respectively (Pilet et al., 1995; Matamoros et al., 2009a). The target strain L. monocytogenes RF191 was isolated from tropical CPS (PFI Nouvelles Vagues, Boulogne-sur-Mer, France). All the strains were stored at $-80^{\circ} \mathrm{C}$ in their culture media with $20 \%$ glycerol (Panreac, Barcelona, Spain). For all experiments, $C$. divergens and $L$. piscium were sub-cultured individually in Elliker broth (Biokar Diagnostic, Beauvais, France) and L. monocytogenes in Brain Heart Infusion (BHI) (Biokar Diagnostic) for $24 \mathrm{~h}$ at $26^{\circ} \mathrm{C}$. After $24 \mathrm{~h}$ of culture, the strains reached their maximal concentrations, $10^{9}$

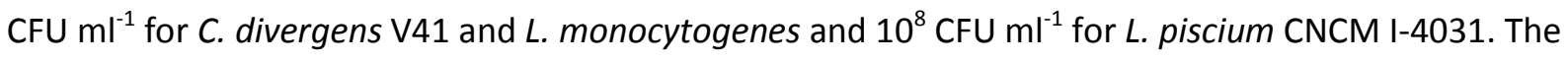
cultures were diluted in physiological saline solution $(0.85 \% \mathrm{NaCl}, 0.1 \%$ tryptone) (Biokar Diagnostic) to achieve an appropriate inoculation solution. 


\subsection{Shrimp and challenge tests}

About $12 \mathrm{~kg}$ of tropical peeled shrimp Penaeus vannamei from Thailand (size 71/90) was cooked by the manufacturer at $71^{\circ} \mathrm{C}$ for 2 min (Miti, Nantes, France) and directly transported to the laboratory under refrigerated conditions. In the first trial, the bioprotective effect of the strains on the quality of tropical CPS was evaluated. Four batches were prepared: (1) untreated shrimp (control); (2) inoculation with $L$. piscium CNCM I-4031 alone; (3) inoculation with C. divergens V41 alone and (4) inoculation with a cocktail of $C$. divergens V41 and L. piscium CNCM I-4031. Shrimp were inoculated at $2 \%(\mathrm{v} / \mathrm{w})$ by spraying diluted cultures to obtain an estimated final level of $5 \times 10^{5} \mathrm{CFU} / \mathrm{g}$ for $C$. divergens V41 and L. piscium CNCM I-4031. No physiological saline solution was added to the control to achieve the same condition as in industry. The spray method was chosen because this method is already used by a shrimp industry based in Nantes (France). After inoculation, each batch was divided into 18 punnets, each containing a 120-g portion of shrimp, and packed under MAP $\left(50 \% \mathrm{CO}_{2}\right.$ and $\left.50 \% \mathrm{~N}_{2}\right)$ as in the French industry (Multivac T250, Wolfertschwenden, Germany). All punnets were stored at $8^{\circ} \mathrm{C}$ for 28 days. Sensory, chemical and microbial analyses were carried out after $0,7,10,14,21$ and 28 days in triplicate (3 punnets). In a second trial one month later, with a new batch of $12 \mathrm{~kg}$ of shrimp, the bioprotective effect of the strains on L. monocytogenes was evaluated. Four batches were prepared: (5) CPS inoculated with $L$. monocytogenes RF191 at a final concentration of $10^{2} \mathrm{CFU} / \mathrm{g}$; (6) inoculation with L. monocytogenes RF191 and L. piscium CNCM I-4031 (10 ${ }^{2}$ and $5 \times 10^{5} \mathrm{CFU} / \mathrm{g}$, respectively); (7) inoculation with $L$. monocytogenes RF191 and C. divergens V41 ( $10^{2}$ and $5 \times 10^{5} \mathrm{CFU} / \mathrm{g}$, respectively) and (8) inoculation with L. monocytogenes RF191, C. divergens V41 and L. piscium CNCM I-4031 $\left(10^{2}, 5 \times 10^{5}\right.$ and $5 \times 10^{5} \mathrm{CFU} / \mathrm{g}$, respectively). After inoculation, each batch was divided into 21 punnets of 120 -g portions and packed under $\operatorname{MAP}\left(50 \% \mathrm{CO}_{2}\right.$ and $\left.50 \% \mathrm{~N}_{2}\right)$. All punnets were stored at $8^{\circ} \mathrm{C}$ for 28 days. Chemical $(\mathrm{pH})$ and 
microbial analyses were carried out after $0,3,7,10,15,21$ and 28 days. All the analyses were done in triplicate and the results presented as the mean value \pm a confidence interval of $95 \%$.

\subsection{Microbial enumeration}

For each day of analysis, 3 punnets per batch were analyzed. 20-g portions (first trial) or 30-g portions (second trial) of shrimp were stomached (Stomacher 400 circulator, Seward Medical, London, UK) for 2 min with 80 and $120 \mathrm{ml}$, respectively, of chilled sterile physiological saline solution with $1 \%$ Tween 80 (Grosseron, Saint-Herblain, France). $0.1 \mathrm{ml}$ of appropriate 10 -fold dilutions was spread on different selective agar plates. For Enterobacteriaceae, $1 \mathrm{ml}$ was pour plated. The microorganisms, culture medium and incubation conditions are listed in Table 1. Anaerobic conditions were performed in anaerobic jars with Anaerocult A and Microbiology Anaerotest (Merck, Darmstadt, Germany). In the cocktail of protective bacteria, C. divergens was enumerated on Elliker agar at $30^{\circ} \mathrm{C}$ as L. piscium cannot grow at this temperature (Matamoros et al., 2009a). L. piscium was enumerated on Elliker agar at $8^{\circ} \mathrm{C}$ where $C$. divergens developed less rapidly than L. piscium and the morphology of the colonies was different. However, the endogenous LAB could not be distinguished from the inoculated bioprotective strains, except that during the first days of the experiment, their concentration was less than inoculated LAB. The detection threshold was 0.7 and $1.7 \mathrm{Log}$ (CFU/g) for Enterobacteriaceae and the other counts, respectively.

\subsection{Physicochemical parameters}

The $\mathrm{pH}$ was measured in the stomached flesh immediately after microbiological analysis by immersing the electrode of the pH-meter (Mettler Toledo AG, Schwerzenbach, Switzerland). Total Volatile Basic Nitrogen (TVBN) was quantified by the Conway micro-diffusion method (Conway and Byrne, 1933)

\subsection{Sensory analysis}

A quantitative descriptive analytical method was used, with a conventional profiling performed by an internal, regularly trained panel of Ifremer, as described by Macé et al. (2014). Panelists (12 people) 
experienced in seafood evaluation were specifically trained on tropical CPS (Fall et al., 2012; Jaffrès et al., 2009; Macé et al., 2014). This analysis was carried out on batches $1,2,3$, and 4, without $L$.

monocytogenes. The panelists first evaluated the overall spoilage level on a continuous scale from 0 to 10 , just after opening the punnet and then 30 s later ( 0 corresponded to not spoiled and 10 to strongly spoiled products). They then scored the following odor descriptors: rice/crustacean, diacetyl/butter, pyrrolidine, floor cloth, sour, acid, fermented, and sulfur. For acceptable samples (global odor score $<5$ 6), the panelists also scored the texture (crunchy, pasty, humidity) and flavor (rice/crustacean, floor cloth, sour, acid, fermented and bitter).

A two-way analysis of variance (ANOVA) was applied to the panelists' scores for spoilage intensity with products (strain) and panelists as independent factors. Significant differences between means were determined using Duncan's multiple range test $(p<0.05)$ (Fizz software, Biosystèmes, Couternon, France).

\subsection{Temporal Temperature Gradient Gel Electrophoresis (TTGE) analysis}

The suspension prepared for bacteriological analysis was used to obtain molecular fingerprints from the control and shrimp matrix inoculated with protective cultures. The DNA was then extracted using the method described previously by Macé et al. (2012).

Bacterial DNA from the inoculated shrimp matrix was analyzed by PCR-TTGE. The V3 region of the $16 \mathrm{~S}$ rRNA gene (194 bp) was PCR-amplified as described by Jaffrès et al. (2009).

The PCR products obtained were subjected to TTGE gel analysis as described previously by Macé et al. (2012). Standardization, analysis and comparison of TTGE fingerprints were monitored using BioNumerics Software, version 7.1 (Applied Maths NV, Sint-Martens-Latem, Belgium) as described by Macé et al. (2012). Experimental fingerprint data were compared to an internal fingerprint database established from bacterial species isolated from marine products. 


\section{Results}

\subsection{Microbiota of naturally contaminated shrimp}

Fig. 1 represents the kinetics of total mesophilic microflora, mesophilic and psychrotrophic LAB,

Enterobacteriaceae and B. thermosphacta of naturally contaminated PCS (control) during MAP storage at $8^{\circ} \mathrm{C}$. The initial contamination level was low $(1.8 \pm 0.1 \mathrm{Log}(\mathrm{CFU} / \mathrm{g})$ for the total mesophilic count and less than the detection level for the other counts. All the microbial groups reached their maximum count in 14 days, except $B$. thermosphacta which was never detected. The highest growth rates and maximum counts were observed for psychrotrophic LAB, whereas mesophilic LAB grew more slowly but reached the same final count after 14 days (8-9 Log (CFU/g)). The colonies in ELK at $8^{\circ} \mathrm{C}$ were small mucoid and opaque and composed of Gram-positive, catalase- and oxidase-negative bacteria, probably LAB producing a polysaccharide. The Enterobacteriaceae count was always 1-2 Log (CFU/g) lower (statistical difference, $P<0.05)$. TTGE fingerprints of the $V 3$ region of $16 \mathrm{~S}$ rRNA genes were performed to evaluate the evolution of the dominant bacterial groups. The results are shown in Fig. 2 . At day 0,5 bands with a very weak intensity were observed. One band (c) was assigned to Carnobacterium/Psychrobacter and 4 bands ( $n 1$ to $n 4$ ) were not assigned. After 7 days, the fingerprints of $n 1, n 2, n 3$ and $n 4$ disappeared whereas fingerprints of Carnobacterium/Psychrobacter intensified until the end of storage. Three fingerprints ( $\mathrm{sb}$ and $\mathrm{sp}$ assigned to Shewanella sp., respectively, and unassigned band $\mathrm{n} 5$ ) appeared at day 7 and persisted until the end of storage. Additionally an unassigned band (n6) appeared at days 21 and 28.

\subsection{Implantation of the bioprotective LAB strains in shrimp}

In batch 2, L. piscium was inoculated at $5.9 \pm 0.2 \mathrm{Log}$ (CFU/g), very close to the desired level (data not shown). The concentration on ELK at $8^{\circ} \mathrm{C}$ reached $8.7 \pm 0.0 \mathrm{Log}(\mathrm{CFU} / \mathrm{g})$ in 14 days and then remained constant. The colony had the same morphology as L. piscium in pure culture and no mucoid colony was present. Moreover, the TTGE results showed intense bands assigned to $L$. piscium. This result confirmed 
that L. piscium was the dominating psychrotrophic LAB throughout storage (band Ip, Fig. 2). In batch 3, the inoculation level of $C$. divergens was $5.4 \pm 0.1 \mathrm{Log}(\mathrm{CFU} / \mathrm{g})$ and the count on ELK at $30^{\circ} \mathrm{C}$ reached $9.5 \pm$ $0.0 \mathrm{Log}$ (CFU/g) in 7 days and until the end of storage (data not shown). This count was always 1 Log (CFU/g) higher than the count on ELK at $30^{\circ} \mathrm{C}$ in batch 1 . The morphology of the colonies looked like $C$. divergens in pure culture. These results indicated that the bioprotective strain dominated the total LAB count in batch 3 . This was confirmed by the TTGE profiles (band $\mathrm{cd}$, assigned to $C$. divergens bands, Fig. 2). In batch 4 , the initial L. piscium and C. divergens counts were $5.8 \pm 0.2 \mathrm{Log}$ (CFU/g) and $5.3 \pm 0.1 \mathrm{Log}$ (CFU/g), respectively. C. divergens was able to grow on ELK at $8^{\circ} \mathrm{C}$ but the colonies could easily be distinguished (white colonies of 1-2 $\mathrm{mm}$ and $0.5 \mathrm{~mm}$ in diameter for L. piscium and C. divergens, respectively). Throughout storage, the counts of presumptive $L$. piscium and $C$. divergens were exactly similar to the counts of pure culture in batches 2 and 3 (data not shown). Therefore, we could conclude that there was no competition between the strains. C. divergens was 1 Log (CFU/g) higher than L. piscium after 7 days and until 28 days. This was confirmed by the TTGE fingerprints (Fig. 2) in which the bands assigned to both protective cultures were present.

\subsection{Effect of the protective cultures on the microbial ecosystem of shrimp}

B. thermosphacta was never detected in the control and biopreserved CPS. Fig. 3 represents the growth kinetics of the Enterobacteriaceae in the different batches of CPS. Enterobacteriaceae grew from $0.7 \pm$ 0.2 to $6.0 \pm 0.3$ at day 7 and $7.1 \pm 0.8 \mathrm{Log}(\mathrm{CFU} / \mathrm{g})$ at day 14 and then remained constant. In the presence of L. piscium (batch 2), no inhibition of the Enterobacteriaceae count was noticed, except at day 28 when the count was 1.0 Log (CFU/g) lower (statistical difference $\mathrm{P}<0.05$ ). With C. divergens (batch 3), a significant inhibition of Enterobacteriaceae of 2-3 Log (CFU/g) was observed, which began at the start of storage and persisted until day 28. The results were similar in batch 4 so it was concluded that the presence of $L$. piscium did not modify the inhibitory effect of $C$. divergens. 
The effect of the protective strains was also determined with the TTGE fingerprints. No strong differences were observed between the fingerprints of the control and CPS inoculated with L. piscium (batch 2), except at day 7 when most of the bands observed in batch 2 were not detected. At day 0, 3 bands were observed (Ip assigned to $L$. piscium as well as the unassigned bands $n 7$ and $n 8$ ). The increase in intensity of the band assigned to L. piscium showed the good implantation of this strain in the shrimp matrix. The unassigned bands $\mathrm{n} 7$ and $\mathrm{n} 8$ totally disappeared after day 7 . After 10 days, the same group of bacteria was found in batches 1 and 2 (sp, sb, c, n5 and n6) throughout storage but with a slightly weaker intensity in batch 2 . It is difficult to know if this was due to a weaker concentration of these species in batch 2 compared to the control. In fact, in batch 2, L. piscium was present in high number and species representing less than $1 \%$ of the total community are not visible in TTGE profiles. In batch 3 , at day 0 , the cd band assigned to $C$. divergens was present and increased immediately after 7 days. Except for the band sb corresponding to Shewanella sp. visible at day 28 but with a very weak intensity, no other band could be detected, proving that $C$. divergens inhibited many of the endogenous bacteria naturally present in CPS. The fingerprints of batches 3 and 4 were similar, except that the band corresponding to the inoculated $L$. piscium was present. The intensity of the band assigned to $C$. divergens fingerprint was higher than that of L. piscium confirming the results obtained by cultural analysis. To conclude, L. piscium had a small effect on the microbial diversity of CPS except at day 7 while C. divergens reduced this biodiversity. The co-culture of $C$. divergens/L. piscium had the same inhibitory capacity as $C$. divergens alone.

In the second trial, in which samples were inoculated with L. monocytogenes, the total ecosystem was once again analyzed and all the results obtained in the first trial were confirmed. Inhibition of Enterobacteriaceae was observed in batches 7 and 8, although it was a little weaker (1 to 2 Log (CFU/g), data not shown). The effect on the microbial diversity of CPS studied by TTGE fingerprints was very weak 
with $L$. piscium whereas $C$. divergens alone or in co-culture inhibited most of the dominating bacteria naturally present in CPS (data not shown).

\subsection{Chemical analysis}

The initial $\mathrm{pH}$ value of CPS was $6.65 \pm 0.05$. In batches 1,3 and 4 , the $\mathrm{pH}$ remained stable throughout storage (data not shown) and no significant difference was observed. In batch 2 with L. piscium alone, acidification to $6.22 \pm 0.05$ was observed in the first 7 days, but after 14 days the $\mathrm{pH}$ increased and reached the same level as in the other batches (6.72 \pm 0.01 at day 28$)$ (data not shown). TVBN in CPS (batch 1) was very low until day $7(<10 \mathrm{mg}-\mathrm{N} / 100 \mathrm{~g})$ but reached high concentrations at days 10 and 14 (40 and $60 \mathrm{mg}-\mathrm{N} / 100 \mathrm{~g}$, respectively, data not shown). L. piscium totally prevented this formation for 10 days. In the presence of $C$. divergens, alone or in co-culture, TVBN production ( $50 \mathrm{mg}-\mathrm{N} / 100 \mathrm{~g}$ ) was observed from 7 days, leading to a much higher concentration than in batches 1 and 2 . At day 14 and until the end of storage, the concentrations were similar in all batches.

\subsection{Effect of the protective cultures on the sensory characteristics of shrimp}

At day 0 , all the samples were considered of very high quality by the panelists, based on the odor, flavor, texture and color evaluation. The presence of the inoculated protective bacteria was not detected.

\subsubsection{Odor characteristics}

Fig. $4 \mathrm{~A}$ represents the global spoilage scores of CPS based on odors during MAP storage at $8^{\circ} \mathrm{C}$. After 7 days, the control was considered not-spoiled with a score of 1.2 on a 10-point scale. However, deterioration occurred rapidly. After 10 and 14 days, the score ranged between 4 and 5 (still acceptable) but after 21 days samples were rejected by the panelists with a score higher than 7 . In the presence of $L$. piscium (batch 2), a significant improvement in quality was noticed until day 10 with a score of 2.7, and samples were also acceptable until fourteen days. Then, the global spoilage score was similar to the control and samples were considered unacceptable for consumption. The samples inoculated with $C$. divergens alone (batch 3) or in co-culture with L. piscium (batch 4) had a score similar to the control until 
day 14 . Then, $C$. divergens alone or in combination with $L$. piscium prevented off-odor formation until day 28 and the final scores were 4.8 and 6.0 in batches 3 and 4, respectively (no statistical difference between batches 3 and 4).

The principal component analysis (PCA) represented in Fig. 5A provides more information on the odor characteristics. Samples and their odor descriptors were projected on the first plane of the PCA, which restored $62.4 \%$ of the total information. The first axis ( $42.7 \%$ of information) enabled the separation of the non-spoiled (right part of Fig. 5A) and lightly/strongly spoiled (left part) samples. Lightly spoiled (upper part) and strongly spoiled (lower part) samples were discriminated on the second axis (19.7\% of information). The control sample at 7 days was considered non-spoiled with a rice odor, typical of freshly cooked tropical shrimp. At 10 and 14 days, the control was lightly spoiled with pyrrolidine, acid, floor cloth and sour odors but still with a rice odor, with a score of around 2 for each descriptor. After 21 days, the control was highly spoiled with strong sulfur, sour and fermented odors (scores from 3 to 5 for these attributes, data not shown). In the presence of L. piscium, the samples were considered non-spoiled at 7 and 10 days with a characteristic rice odor (intensity of 3.7 and 3.3, respectively), showing a prevention of the sensory deterioration of CPS during 10 days. After 14 days, the samples were considered lightly spoiled like the control but the odors were different and less unpleasant (rice and diacetyl/butter odor with an intensity of 2.1 and 1.4, respectively). At days 20 and 28, the off-odor characteristics were similar in batches 1 and 2 . The results in Fig. $5 \mathrm{~A}$ clearly show that batches 3 and 4 inoculated with $C$. divergens alone or in co-culture remained in the upper-left part of the figure and never became highly spoiled. The descriptors were pyrrolidine, floor cloth, sour and fermented for batch 3 and diacetyl/butter for batch 4 . It is important to note that until 7 days, the presence of $C$. divergens was responsible for slight off-odor release whereas in association with L. piscium (batch 4) these odors were not detected and the sample was very similar to the control (i.e. good quality). 


\subsubsection{Flavor characteristics}

Fig. 4B represents the global spoilage scores based on flavor and texture evaluation of CPS until 14 days of storage. After 21 days, the panelists did not consume the products because of strong off-odors in the control. After 7 days, the control was of very high quality with a score of 0.6 . Then it increased to reach 3.1 after 14 days. L. piscium significantly prevented the spoilage of CPS. The global flavor/texture scores were 1.2 and 1.6 points lower than the control after 10 and 14 days, respectively, and never exceeded the score of 1.3. After 7 days, and until 10 days to a lesser extent, the presence of $C$. divergens was detected by the panelists and the spoilage score was higher than in the control (3.3 vs. 0.6 at day 7 ). After 14 days (and probably more if the products had been eaten), the beneficial effect of $C$. divergens against the spoilage of CPS (batch 1) was evidenced. Interestingly, the presence of L. piscium significantly prevented the off-flavors released by $C$. divergens. In fact, in co-culture, the spoilage score was 1.7 and 1.5 after 7 and 10 days, respectively, and 3.3 and 2.4 with $C$. divergens alone. At day 14 , the effect of $C$. divergens alone or in co-culture was similar, as for the odors. The PCA summarizing the main flavor and texture characteristics is represented in Fig. 5B. The first axis restored $58.8 \%$ of the total information and enabled the separation of the non-spoiled (left part of Fig. 5B) and lightly spoiled (right part) samples. The second axis ( $18.6 \%$ of information) discriminated samples with sour and fermented flavors (upperright part of the plan) from those with a pasty texture and floor cloth and bitter taste (lower-right part). The non-spoiled samples were characterized by a rice/crustacean flavor and crispy texture as were the control at day 7, all samples of batch 2 (in the presence of L. piscium) and samples inoculated with $C$. divergens and L. piscium at day 7. All the other samples were lightly spoiled, mainly with a pasty texture and floor cloth and bitter tastes. Samples of batch 3 inoculated with $C$. divergens had a specific signature of a sour and fermented taste at the beginning of the experiment. In conclusion, as for the odors until 14 days, L. piscium had the main positive effect on the quality of CPS. The unpleasant taste of $C$. divergens until 10 days of storage disappeared in the presence of $L$. piscium. 


\subsection{Effect of the protective cultures on L. monocytogenes}

Fig. 6 shows the growth kinetics of L. monocytogenes alone in CPS (control) and in the presence of $L$. piscium, C. divergens and the two strains simultaneously. L. monocytogenes increased rapidly from an initial level of $2.3 \pm 0.1$ to $7.5 \pm 0.2 \mathrm{Log}(\mathrm{CFU} / \mathrm{g})$ at day 10 , then decelerated to $8.1 \pm 0.1 \mathrm{Log}$ (CFU/g) at 14 days and stabilized until the end of storage. In the presence of L. piscium (batch 6), a statistical inhibition of 1-2 Log (CFU/g) was observed throughout storage. In the presence of $C$. divergens (batch 7) and a mixture of the two protective LAB strains (batch 8), the growth of L. monocytogenes was totally prevented for 10 days (at day 7, the L. monocytogenes count was even lower than the detection threshold). Then L. monocytogenes grew at the same rate as when alone in batch 6 and reached $6.0 \pm 0.5$ and $6.6 \pm 0.2 \mathrm{Log}$ (CFU/g) in batches 7 and 8, respectively, after 28 days, corresponding to a 2 Log (CFU/g) diminution. No statistical difference was observed between the L. monocytogenes counts in batches 7 and 8 . No additional or synergetic effect was noticed between L. piscium and C. divergens

\section{Discussion}

Our results confirmed that the microbial ecosystem of tropical CPS is complex and can reach 8-9 Log (CFU/g) in a few days at $8^{\circ} \mathrm{C}$ under MAP. The heat treatment of shrimp reduces the initial contamination but does not eliminate all the microorganisms, probably because shrimp are not gutted. In addition, the various processing and handling steps and environmental conditions in the industry play a role in the recontamination. The different microbial counts revealed that psychrotrophic and mesophilic LAB dominated the ecosystem, with the presence of $C$. divergens revealed by TTGE fingerprints. Many authors, using culture and culture-independent techniques, have reported the importance of LAB such as Carnobacterium (divergens, maltaromaticum, jeotgali, inhibens, funditum, alterfunditum, mobile), Enterococcus (faecalis, faecium), Vagococcus (penaei, fluvialis), Leuconostoc (gasicomitatum, mesenteroides), Weissella viridescens and Streptococcus parauberis in cooked and peeled $P$. vannamei 
stored under $\mathrm{CO}_{2} / \mathrm{N}_{2}$ (50/50) (Chaillou et al., 2015; Jaffrès et al., 2009, 2010). The predominance of LAB, frequently including Carnobacterium, has also been reported in other species of arctic or tropical shrimp under various storage conditions (Calliauw et al., 2016; Dabade, 2015; Jaffrès et al., 2009; Mejlholm et al., 2005). The role of Carnobacterium in spoilage depends on the species and strains but some $C$. divergens and C. maltaromaticum have produced chlorine, cheese/feet, sour and butter off-odors in peeled Pandalus borealis and P. vannamei (Jaffrès et al., 2011; Laursen et al., 2006; Mejlholm et al., 2005). Although the count of Enterobacteriaceae was high (7 Log (CFU/g)), it is important to note that the most frequently found species Serratia liquefaciens/proteamaculans (Jaffrès et al., 2009) was not evidenced in this study by TTGE profiling. It is possible that their count was too low to be detected by TTGE. Moreover, the selectivity of VRBG is questionable. In a study performed on tuna loins, herring fillets and hot-smoked mackerel by Podeur et al. (2015), all the colonies isolated from VRBG were Pseudomonas/Shewanella-like. In this study, the DNA fingerprints of Shewanella spp. were present in the control CPS with a high intensity of the bands. These species are known as strong spoilers of some vacuum-packed fish (Gram and Huss, 1996; Vogel et al., 2005) and for their production of trimethylamine and $\mathrm{H}_{2} \mathrm{~S}$ components. Shewanella spp. have been isolated from CPS in our laboratory in the framework of a national project MIPROMER (unpublished data) and are part of the dominant microbiota of Crangon crangon (Calliauw et al., 2016). S. baltica, inoculated at high level in CPS, has clearly been pointed out as a major spoiler, with sour and cabbage/sulfur odors (Macé et al., 2014). The TVBN concentration in CPS was very high. Different authors have reported the production of up to $90 \mathrm{mg}-\mathrm{N} / 100 \mathrm{~g}$ by $C$. maltaromaticum, C. divergens and $V$. penaei (challenge tests in artificially inoculated shrimp, 3 weeks at $8^{\circ} \mathrm{C}$ under MAP) as well as 60,50 and $40 \mathrm{mg}-\mathrm{N} / 100 \mathrm{~g}$ for S. baltica, S. liquefaciens and B. thermosphacta, respectively (Jaffrès et al., 2011; Macé et al., 2014).

L. piscium CNCM I-4031 inoculated in shrimp grew very well, as already shown by Fall et al. (2010a, 2010b; 2012,). Its presence prevented the sensory deterioration of CPS for 14 days compared to the 
control, but no beneficial effect was observed after 21 days. These results were correlated with the TVBN content, which was lower in batch 2 for 10 days. In the study of Matamoros et al. (2009b), the bioprotective effect of $L$. piscium was more pronounced, with a shelf-life extended to 28 days. The hypothesis was that $L$. piscium had inhibited the growth of $B$. thermosphacta, which is a well-known spoilage bacteria of shrimp (Jaffrès et al., 2011; Laursen et al., 2006). In fact, B. thermosphacta inoculated in sterile shrimp spoiled the products after 11 days at $8^{\circ} \mathrm{C}$ under MAP, whereas in the presence of $L$. piscium, the samples were still acceptable after 38 days (Fall et al., 2012). In the present study, B. thermosphacta was absent in the control. The improvement in the sensory quality may be due to an inhibition of Carnobacterium or Shewanella spp., for which the intensity of the assigned bands was weaker in batch 2 than in the control. However, the antimicrobial effect of another protective strain of $L$. piscium (EU2229) against a mixture of S. baltica, B. thermosphacta and C. maltaromaticum in CPS confirmed the high sensitivity of $B$. thermosphacta whereas C. maltaromaticum was slightly inhibited (1 Log (CFU/g) and S. baltica was not (unpublished data from our laboratory).

C. divergens $\mathrm{V} 41$ grew rapidly in CPS and reached a final concentration $1 \mathrm{Log}$ (CFU/g) higher than $L$. piscium CNCM I-4031. In contrast with L. piscium, this strain was detected by the panelists in the first 10 days of storage, with pyrrolidine, floor cloth, sour and fermented odors and taste, when the control (batch 1) was still of good quality. Slight off-odors or a bitter flavor have already been detected in sterile blocks of CSS inoculated with C. divergens V41 (Brillet et al., 2005). Very interestingly, the presence of $L$. piscium (batch 4) reduced this negative perception at the early stage of storage. However, the concentration of TVBN with the cocktail was the same as with $C$. divergens alone. This indicates that compounds other than nitrogen-containing molecules are involved in spoilage, as demonstrated in naturally contaminated cooked shrimp by Dalgaard and Jørgensen (2000) and Mejlholm et al. (2005) and for raw peeled royal shrimp Penaeus kerathurus (Sadok et al., 2004). From day 14 and until the end of storage, $C$. divergens prevented the off-odor formation observed in the control. The presence of $L$. 
piscium did not reduce this protective effect. To our knowledge, this is the first time that an activity of Carnobacterium against seafood spoilage has been reported. In CSS for example, C. divergens V41 did not extend the sensory shelf-life (Brillet et al., 2005). The beneficial effect may be due to the inhibition of bacteria enumerated in VRBG (2 to 3 Log (CFU/g)). Furthermore, TTGE analysis clearly showed the extinction of many bands at 7 days and after.

Concerning L. monocytogenes, an inhibition of $1 \mathrm{Log}$ (CFU/g) was observed after day 7 in the presence of L. piscium. This is lower than the 3-4 Log (CFU/g) observed by Fall et al. (2010b). In the latter study, the strains were inoculated in shrimp sterilized by ionization whereas in the former, CPS contained its own endogenous microbiota. As the mechanism involved in this inhibition is cell-to-cell contact-dependent (Saraoui et al., 2016), the presence of endogenous bacteria in CPS at high concentration may prevent physical contact between L. piscium and L. monocytogenes. However, Matamoros et al. (2009b) obtained a 2 Log (CFU/g) reduction by challenge tests in naturally contaminated shrimp. C. divergens had a listericidal effect that lasted 7-10 days. Then L. monocytogenes grew at approximately the same rate as in the control but the count was always 2 to 5 (CFU/g) lower. C. divergens V41 produces a very active class Ila bacteriocin, divercin V41 (Metivier et al., 1998). In sterile CSS blocks, C. divergens V41 totally prevented the growth of $L$. monocytogenes for 28 days under vacuum packaging at $4-8^{\circ} \mathrm{C}$ (Brillet et al., 2004), and it was proved, using a divercin- mutant, that the inhibition of L. monocytogenes was specifically due to this bacteriocin (Richard et al., 2003). This difference may be explained by the fact that the target $L$. monocytogenes strain, the matrix and the packaging conditions were different as well as by the presence of naturally contaminating bacteria, which can also produce proteases active against bacteriocin. Some spoilage LAB may be sensitive to divercin, which may explain the improvement in the sensory quality of CPS with $C$. divergens. In the co-culture of $L$. piscium and $C$. divergens, the inhibition of L. monocytogenes was similar to that with C. divergens alone. There was no additional or synergic effect. 
In conclusion, we have shown that the two protective bacteria do not inhibit each other when coinoculated in CPS. The inhibitory effect of $C$. divergens on L. monocytogenes in naturally contaminated shrimp has been demonstrated for the first time. L. piscium also inhibits L. monocytogenes but there is no additional or synergic effect between the two LAB. C. divergens V41 is very effective at preventing the sensory deterioration of CPS stored under MAP at $8^{\circ} \mathrm{C}$. However, the panelists could detect the presence of $C$. divergens during the first 10 days of storage. L. piscium improves the sensory quality of CPS for 14 days only. In co-culture, L. piscium is very effective at eliminating the off-odors and flavors released by $C$. divergens in the early stage of storage. Therefore, the use of a cocktail of L. piscium CNCM I-4031 and C. divergens $\mathrm{V} 41$ is recommended in a strategy of biopreservation of seafood.

\section{Acknowledgements}

Taous Saraoui was the recipient of a Ph.D. fellowship from the French Ministry of Higher Education and Research. This study was supported by a public grant from the Région Pays de la Loire (France) (project COM-BACT). The authors thank Mireille Cardinal for assistance with sensory analysis and for her advice, Justine Greyo for her help in the experimental tests and Carol Robins for the English language editing of the manuscript.

\section{References}

Brillet, A., Pilet, M.F., Prevost, H., Bouttefroy, A., Leroi, F., 2004. Biodiversity of Listeria monocytogenes sensitivity to bacteriocin-producing Carnobacterium strains and application in sterile coldsmoked salmon. J. Appl. Microbiol. 97, 1029-1037.

Brillet, A., Pilet, M.-F., Prevost, H., Cardinal, M., Leroi, F., 2005. Effect of inoculation of Carnobacterium divergens V41, a biopreservative strain against Listeria monocytogenes risk, on the 
microbiological, chemical and sensory quality of cold-smoked salmon. Int. J. Food Microbiol. 104, 309-324.

Calliauw, F., De Mulder, T., Broekaert, K., Vlaemynck, G., Michiels, C., Heyndrickx, M., 2016. Assessment throughout a whole fishing year of the dominant microbiota of peeled brown shrimp (Crangon crangon) stored for 7 days under modified atmosphere packaging at $4{ }^{\circ} \mathrm{C}$ without preservatives. Food Microbiol. 54, 60-71. doi:10.1016/j.fm.2015.10.016

Calo-Mata, P., Arlindo, S., Boehme, K., Miguel, T. de, Pascoal, A., Barros-Velazquez, J., 2007. Current applications and future trends of lactic acid bacteria and their bacteriocins for the biopreservation of aquatic food products. Food Bioprocess Technol. 1, 43-63.

Chaillou, S., Chaulot-Talmon, A., Caekebeke, H., Cardinal, M., Christieans, S., Denis, C., Hélène Desmonts, M., Dousset, X., Feurer, C., Hamon, E., Joffraud, J.-J., La Carbona, S., Leroi, F., Leroy, S., Lorre, S., Macé, S., Pilet, M.-F., Prévost, H., Rivollier, M., Roux, D., Talon, R., Zagorec, M., ChampomierVergès, M.-C., 2015. Origin and ecological selection of core and food-specific bacterial communities associated with meat and seafood spoilage. ISME J. 9, 1105-1118.

Conway, E.J., Byrne, A., 1933. An absorption apparatus for the micro-determination of certain volatile substances. Biochem. J. 27, 419-429.

Dabade, D.S., 2015. Shrimp quality and safety management along the supply chain in Benin. PhD thesis, Wageningen University, Wageningen, $158 \mathrm{p}$.

Dalgaard, P., Jørgensen, L.V., 2000. Cooked and brined shrimps packed in a modified atmosphere have a shelf-life of $>7$ months at $0{ }^{\circ} \mathrm{C}$, but spoil in $4-6$ days at $25^{\circ} \mathrm{C}$. Int. J. Food Sci. Technol. $35,431-$ 442.

Fall, P.A., Leroi, F., Cardinal, M., Chevalier, F., Pilet, M.F., 2010a. Inhibition of Brochothrix thermosphacta and sensory improvement of tropical peeled cooked shrimp by Lactococcus piscium CNCM I4031. Lett. Appl. Microbiol. 50, 357-361. 
Fall, P.A., Leroi, F., Chevalier, F., Guerin, C., Pilet, M.-F., 2010b. Protective effect of a nonbacteriocinogenic Lactococcus piscium CNCM I-4031 strain against Listeria monocytogenes in sterilized tropical cooked peeled shrimp. J. Aquat. Food Prod. Technol. 19, 84-92.

Fall, P.A., Pilet, M.F., Leduc, F., Cardinal, M., Duflos, G., Guérin, C., Joffraud, J.-J., Leroi, F., 2012. Sensory and physicochemical evolution of tropical cooked peeled shrimp inoculated by Brochothrix thermosphacta and Lactococcus piscium CNCM I-4031 during storage at $8^{\circ} \mathrm{C}$. Int. J. Food Microbiol. 152, 82-90.

FAO, 2012. FAO Yearbook - Fishery and Aquaculture Statistics [WWW Document]. URL http://www.fao.org/3/a-i3740t.pdf (accessed 8.26.15).

FAO-Globefish, 2015a. GLOBEFISH Highlights [WWW Document]. Issuu. URL http://issuu.com/globefish/docs/16_february_gh_online (accessed 11.6.15).

Gandhi, M., Chikindas, M.L., 2007. Listeria: A foodborne pathogen that knows how to survive. Int. J. Food Microbiol. 113, 1-15.

Ghanbari, M., Jami, M., Domig, K.J., Kneifel, W., 2013. Seafood biopreservation by lactic acid bacteria - A review. LWT - Food Sci. Technol. 54, 315-324.

Gram, L., Huss, H.H., 1996. Microbiological spoilage of fish and fish products. Int. J. Food Microbiol., Specific Spoilage Organisms 33, 121-137.

Jaffrès, E., Lalanne, V., Macé, S., Cornet, J., Cardinal, M., Sérot, T., Dousset, X., Joffraud, J.-J., 2011. Sensory characteristics of spoilage and volatile compounds associated with bacteria isolated from cooked and peeled tropical shrimps using SPME-GC-MS analysis. Int. J. Food Microbiol. 147, 195-202.

Jaffrès, E., Prévost, H., Rossero, A., Joffraud, J.-J., Dousset, X., 2010. Vagococcus penaei sp. nov., isolated from spoilage microbiota of cooked shrimp (Penaeus vannamei). Int. J. Syst. Evol. Microbiol. 60, 2159-2164. 
Jaffrès, E., Sohier, D., Leroi, F., Pilet, M.F., Prévost, H., Joffraud, J.J., Dousset, X., 2009. Study of the bacterial ecosystem in tropical cooked and peeled shrimps using a polyphasic approach. Int. J. Food Microbiol., 15th Meeting of the Club des Bactéries Lactiques 131, 20-29.

Jami, M., Ghanbari, M., Zunabovic, M., Domig, K.J., Kneifel, W., 2014. Listeria monocytogenes in aquatic food products -A review. Compr. Rev. Food Sci. Food Saf. 13, 798-813.

Joffraud, J.-J., Cardinal, M., Cornet, J., Chasles, J.-S., Léon, S., Gigout, F., Leroi, F., 2006. Effect of bacterial interactions on the spoilage of cold-smoked salmon. Int. J. Food Microbiol. 112, 51-61.

Laursen, B.G., Leisner, J.J., Dalgaard, P., 2006. Carnobacterium species: effect of metabolic activity and interaction with Brochothrix thermosphacta on sensory characteristics of modified atmosphere packed shrimp. J. Agric. Food Chem. 54, 3604-3611.

Lecuit, M., Lecle, Al., Chenal-Francisque, V., Charlier-Woerther, C., Bracq-Dieve, H., Thouveno, P., Morvan, A., Lavina, M., 2015. Rapport d'activité de l'institut Pasteur vis-à-vis de la surveillance microbiologique de la listériose en France. Paris, France

Leroi, F., Cornet, J., Chevalier, F., Cardinal, M., Coeuret, G., Chaillou, S., Joffraud, J.-J., 2015. Selection of bioprotective cultures for preventing cold-smoked salmon spoilage. Int. J. Food Microbiol. 213, $79-87$

Macé, S., Cardinal, M., Jaffrès, E., Cornet, J., Lalanne, V., Chevalier, F., Sérot, T., Pilet, M.-F., Dousset, X., Joffraud, J.-J., 2014. Evaluation of the spoilage potential of bacteria isolated from spoiled cooked whole tropical shrimp (Penaeus vannamei) stored under modified atmosphere packaging. Food Microbiol. 40, 9-17.

Macé, S., Cornet, J., Chevalier, F., Cardinal, M., Pilet, M.-F., Dousset, X., Joffraud, J.-J., 2012.

Characterisation of the spoilage microbiota in raw salmon (Salmo salar) steaks stored under vacuum or modified atmosphere packaging combining conventional methods and PCR-TTGE. Food Microbiol. 
Matamoros, S., Leroi, F., Cardinal, M., Gigout, F., Kasbi Chadli, F., Cornet, J., Prévost, H., Pilet, M.F., 2009. Psychrotrophic lactic acid bacteria used to improve the safety and quality of vacuum-packaged cooked and peeled tropical shrimp and cold-smoked salmon. J. Food Prot. 72, 365-374.

Matamoros, S., Pilet, M.F., Gigout, F., Prévost, H., Leroi, F., 2009. Selection and evaluation of seafoodborne psychrotrophic lactic acid bacteria as inhibitors of pathogenic and spoilage bacteria. Food Microbiol. 26, 638-644.

Mejlholm, O., Bøknæs, N., Dalgaard, P., 2005. Shelf life and safety aspects of chilled cooked and peeled shrimps (Pandalus borealis) in modified atmosphere packaging. J. Appl. Microbiol. 99, 66-76. Metivier, A., Pilet, M.-F., Dousset, X., Sorokine, O., Anglade, P., Zagorec, M., Piard, J.-C., Marlon, D., Cenatiempo, Y., Fremaux, C., 1998. Divercin V41, a new bacteriocin with two disulphide bonds produced by Carnobacterium divergens V41: primary structure and genomic organization. Microbiology 144, 2837-2844.

Ortolani C, C., Bruijnzeel-koomen, C., Bengtsson, U., Bindslev-jensen, C., Björkstén, B., Høst, A., Ispano, M., Jarish, R., Madsen, C., Nekam, K., Paganelli, R., Poulsen, L., Wüthrich, B., 1999. Controversial aspects of adverse reactions to food. Allergy 54, 27-45.

Pilet, M.-F., Dousset, X., Barré, R., Novel, G., Desmazeaud, M., Piard, J.-C., 1995. Evidence for two bacteriocins produced by Carnobacterium piscicola and Carnobacterium divergens isolated from fish and active against Listeria monocytogenes. J. Food Prot. 58, 256-262.

Pilet, M.-F., Leroi, F., 2011. Applications of protective cultures, bacteriocins and bacteriophages in fresh seafood and seafood products, In: Lacroix, C. (Ed.), Protective Cultures, Antimicrobial Metabolites and Bacteriophages for Food and Beverage Biopreservation, Woodhead Publishing Series in Food Science, Technology and Nutrition. Woodhead Publishing, pp. 324-347.

Podeur, G., Dalgaard, P., Leroi, F., Prévost, H., Emborg, J., Martinussen, J., Hansen, L.H., Pilet, M.-F., 2015. Development of a real-time PCR method coupled with a selective pre-enrichment step for 
quantification of Morganella morganii and Morganella psychrotolerans in fish products. Int. J. Food Microbiol. 203, 55-62.

Richard, C., Brillet, A., Pilet, M. f., Prévost, H., Drider, D., 2003. Evidence on inhibition of Listeria monocytogenes by divercin V41 action. Lett. Appl. Microbiol. 36, 288-292.

Rutherford, T.J., Marshall, D.L., Andrews, L.S., Coggins, P.C., Wes Schilling, M., Gerard, P., 2007. Combined effect of packaging atmosphere and storage temperature on growth of Listeria monocytogenes on ready-to-eat shrimp. Food Microbiol. 24, 703-710.

Sadok, S., Abdelmoulah, A., El Abed, A., 2004. Combined effect of sepia soaking and temperature on the shelf life of peeled shrimp Penaeus kerathurus. Food Chem. 88, 115-122.

Saraoui, T., Fall, P.A., Leroi, F., Antignac, J.-P., Chéreau, S., Pilet, M.F., 2016. Inhibition mechanism of Listeria monocytogenes by a bioprotective bacteria Lactococcus piscium CNCM I-4031. Food Microbiol., 19th Meeting of the Club des Bactéries Lactiques 53, Part A, 70-78. 2

Vogel, B.F., Venkateswaran, K., Satomi, M., Gram, L., 2005. Identification of Shewanella baltica as the most important H2S-producing species during iced storage of Danish marine fish. Appl. Environ. Microbiol. 71, 6689-6697.

Zarei, M., Maktabi, S., Ghorbanpour, M., 2011. Prevalence of Listeria monocytogenes, Vibrio parahaemolyticus, Staphylococcus aureus, and Salmonella spp. in seafood products using multiplex polymerase chain reaction. Foodborne Pathog. Dis. 9, 108-112. doi:10.1089/fpd.2011.0989 
Figure 1: Growth of the endogenous microflora of cooked and peeled shrimp packaged under modified atmosphere and stored at $8^{\circ} \mathrm{C}$ for 28 days. (x) total mesophilic bacteria $(\diamond)$ mesophilic lactic acid bacteria; (০) psychrotrophic lactic acid bacteria; $(\Delta)$ Brochothrix thermosphacta; ( $\square$ ) Enterobacteriaceae

Figure 2: Digitized TTGE profiles of the $16 \mathrm{~S}$ rRNA gene of the V3 region obtained by PCR amplification of bacterial DNA extracted from shrimp. Batch 1 (control): non-inoculated shrimp; batch 2 (Lp): inoculated with Lactococcus piscium CNCM I-4031; batch 3 (Cd): inoculated with Carnobacterium divergens V41; batch $4(\mathrm{Lp}+\mathrm{Cd})$ : inoculated with a co-culture of Carnobacterium divergens V41 and Lactococcus piscium CNCM I-4031. Lanes D0-D28 correspond to samples at days 0, 7, 10, 14, 21, and 28. Bands: "c" represents Carnobacterium divergens, jeotgali or Psychrobacter glacincola, "cd" is C. divergens, "Ip" is $L$. piscium, "sp" is Shewanella putrefaciens, "sb" is Shewanella baltica and "n1 to n8" are bands not assigned to any bacteria in our database. Encircled bands represent the inoculated lactic acid bacteria. Figure 3: Growth of Enterobacteriaceae in cooked and peeled shrimp packed under modified atmosphere and stored at $8^{\circ} \mathrm{C}$ for 28 days. ( $\$$ ) control; ( $\left.\mathbf{\square}\right)$ batch 2 inoculated with Lactococcus piscium CNCM I-4031; ( $\mathbf{\Delta}$ ) batch 3 inoculated with Carnobacterium divergens V41 (•) batch 4 inoculated with Lactococcus piscium CNCM I-4031 and Carnobacterium divergens V41.

Figure 4: Sensory score of cooked and peeled shrimp packed under modified atmosphere and stored at $8^{\circ} \mathrm{C}$ based on $(A)$ odors and $(B)$ flavors and texture, determined by 12 trained panelists. Continuous scale from 0 to 10 , with 0 corresponding to unspoiled and 10 to highly spoiled samples. Different letters indicate significant differences between samples. ( $)$ control; ( $\mathbf{\square})$ batch 2 inoculated with Lactococcus piscium CNCM I-4031; ( $\mathbf{\Delta}$ ) batch 3 inoculated with Carnobacterium divergens V41 ( $\bullet$ ) batch 4 inoculated with Lactococcus piscium CNCM I-4031 and Carnobacterium divergens V41.

Figure 5: Simultaneous representation of samples of cooked and peeled shrimp packed under modified atmosphere and stored at $8^{\circ} \mathrm{C}$ and $(\mathrm{A})$ odor descriptors and (B) flavor and texture descriptors on plane 12 of principal component analysis. Sample nomenclature: 1: control; 2: Lactococcus piscium CNCM I- 
4031; 3: Carnobacterium divergens V41; 4: co-culture of Carnobacterium divergens V41 and Lactococcus piscium CNCM I-4031, D7-D28: days of storage (7, 10, 14, 21, and 28).

Figure 6: Growth of Listeria monocytogenes RF191 in cooked and peeled shrimp packed under modified atmosphere and stored at $8^{\circ} \mathrm{C}$ for 28 days. ( $)$ control; ( $(\mathbf{a})$ batch 2 inoculated with Lactococcus piscium CNCM I-4031; ( $\mathbf{\Delta}$ ) batch 3 inoculated with Carnobacterium divergens V41; (•) batch 4 inoculated with Lactococcus piscium CNCM I-4031 and Carnobacterium divergens V41. 
Table 1: Culture media and conditions used to enumerate the inoculated bacteria and endogenous microflora of cooked and peeled shrimp packaged under modified atmosphere and stored at $8^{\circ} \mathrm{C}$ for 28 days

\begin{tabular}{lll}
\hline Culture medium & Growth conditions & Target strains
\end{tabular}

\begin{tabular}{lll}
\hline Elliker & $48 \mathrm{~h}$ at $30^{\circ} \mathrm{C}$, anaerobic & Carnobacterium divergens and other mesophilic lactic acid \\
& & bacteria \\
Elliker & 5 days at $8^{\circ} \mathrm{C}$, anaerobic & Lactococcus piscium, Carnobacterium divergens and other \\
& & psychrotrophic lactic acid bacteria \\
VRBG & $72 \mathrm{~h}$ at $30^{\circ} \mathrm{C}$, aerobic & Enterobacteriaceae \\
STAA & $48-72 \mathrm{~h}$ at $20^{\circ} \mathrm{C}$, aerobic & Brochothrix thermosphacta \\
PCA & $48 \mathrm{~h}$ at $30^{\circ} \mathrm{C}$, aerobic & Total mesophilic bacteria \\
PALCAM & $48 \mathrm{~h}$ at $37^{\circ} \mathrm{C}$, aerobic & Listeria monocytogenes
\end{tabular}


Figure 1

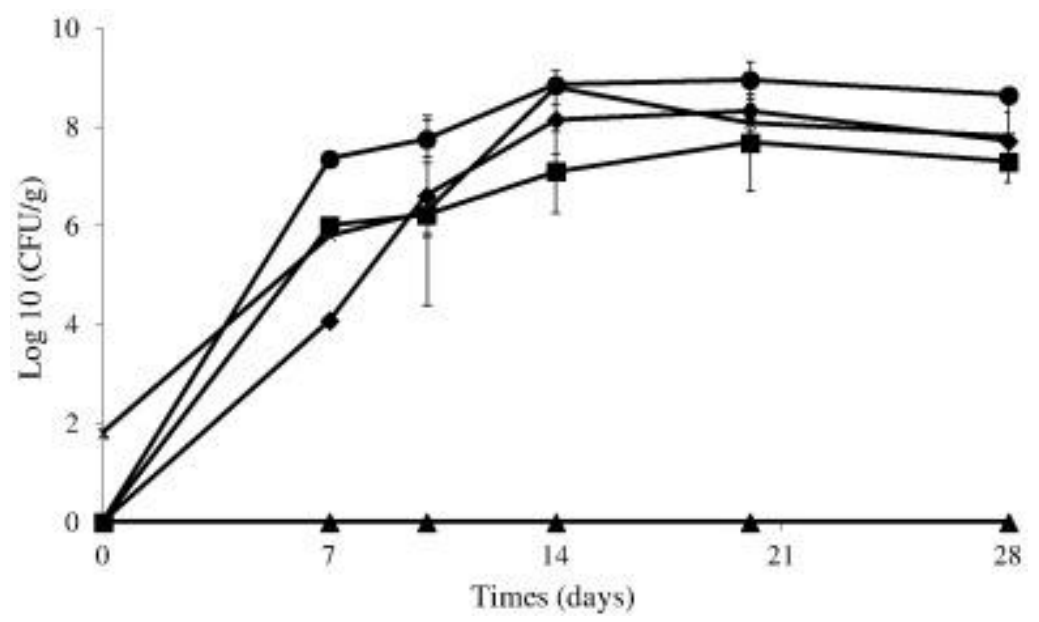

Figure 2

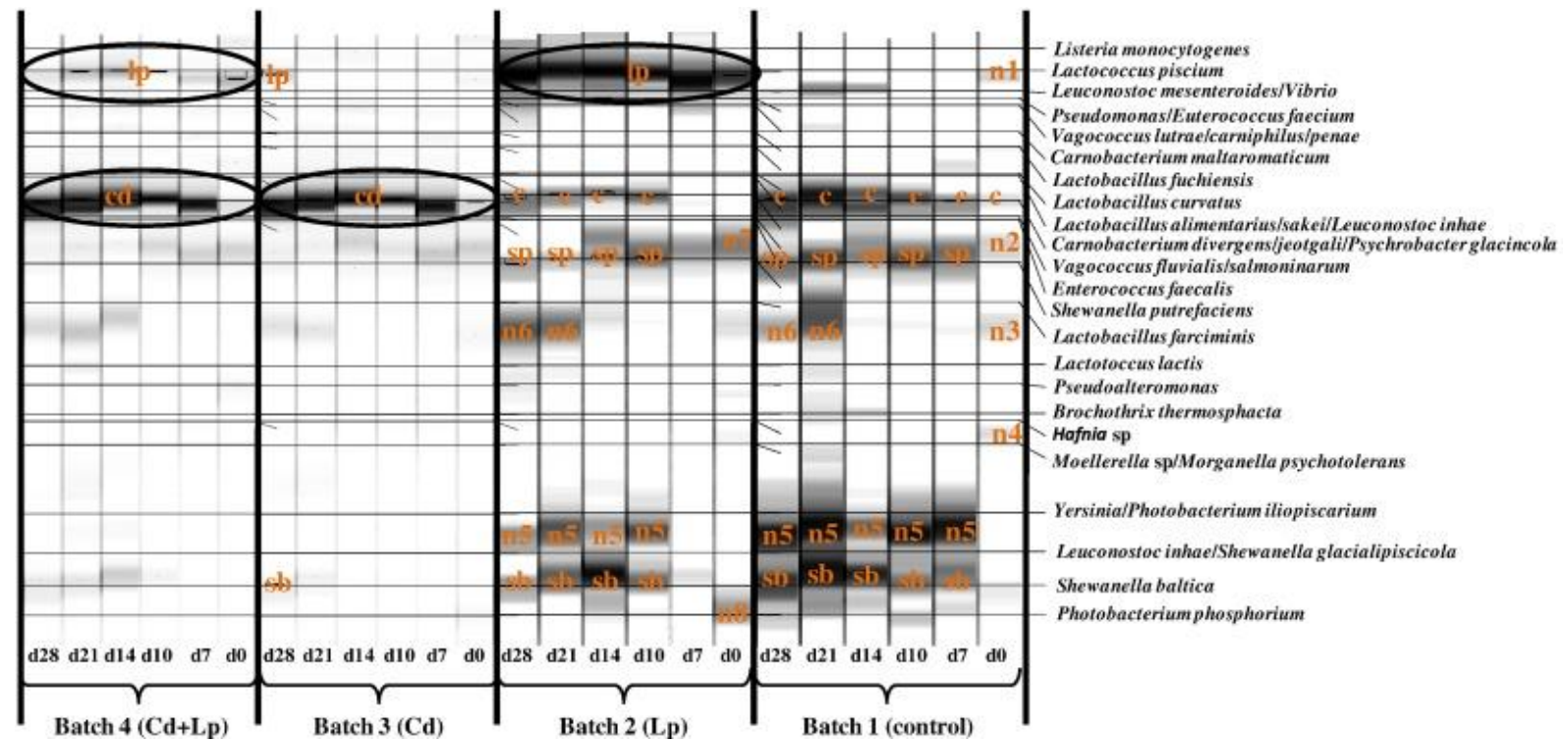


Figure 3

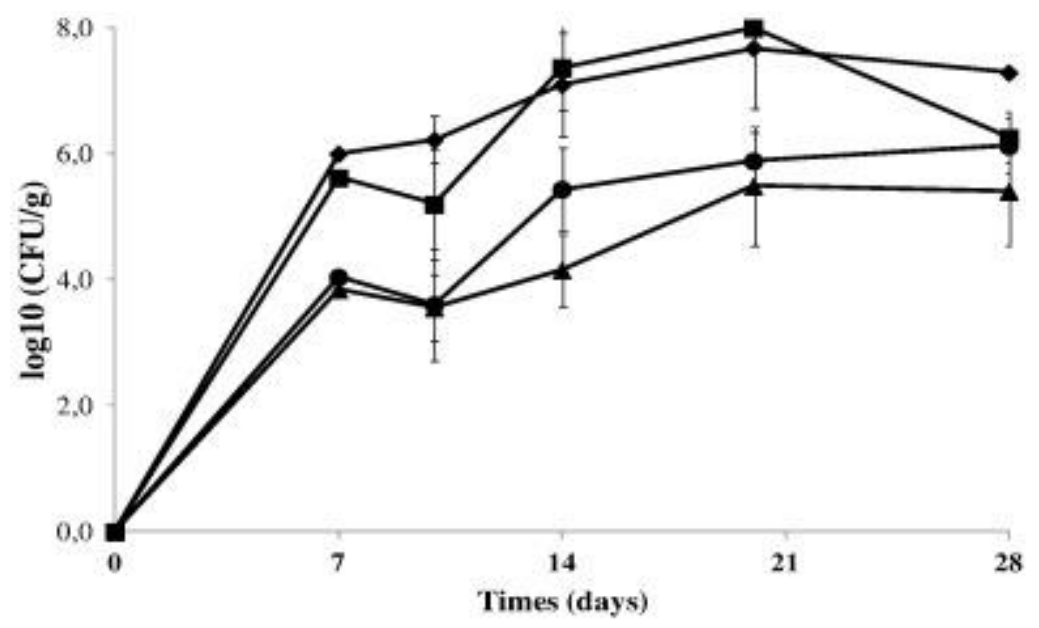

Figure 4

(A)

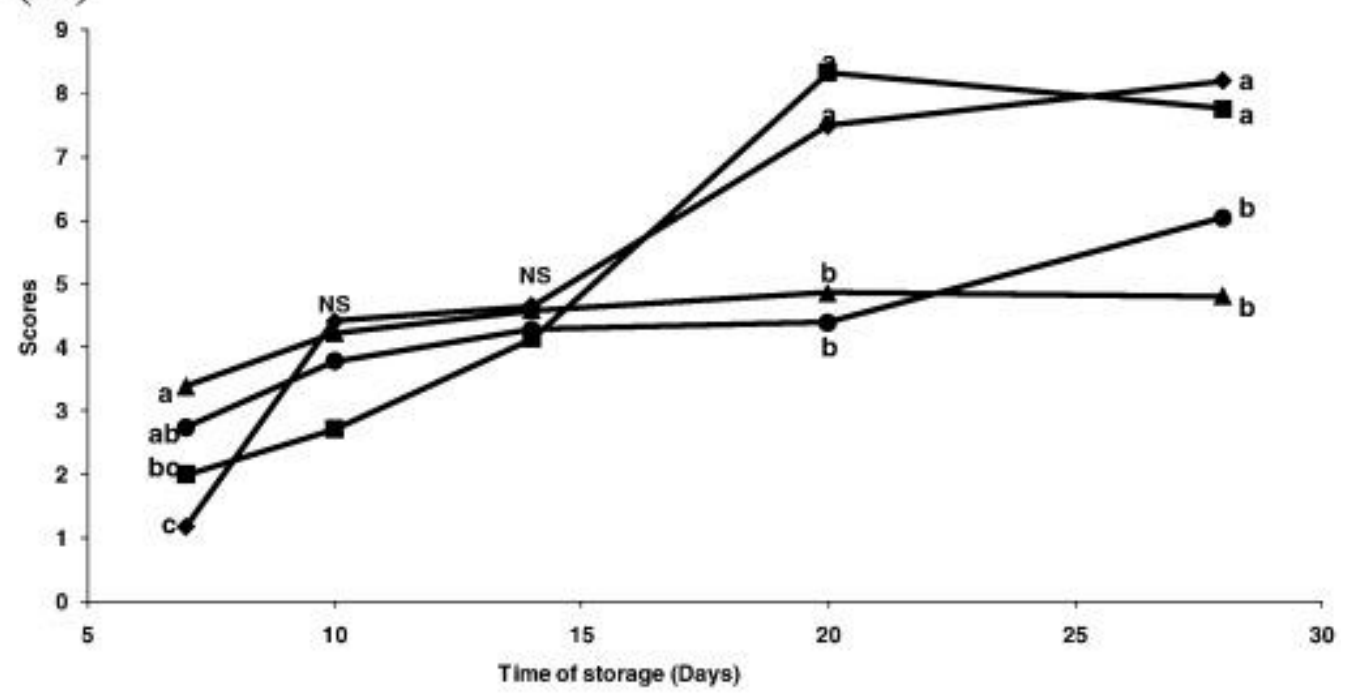

(B)

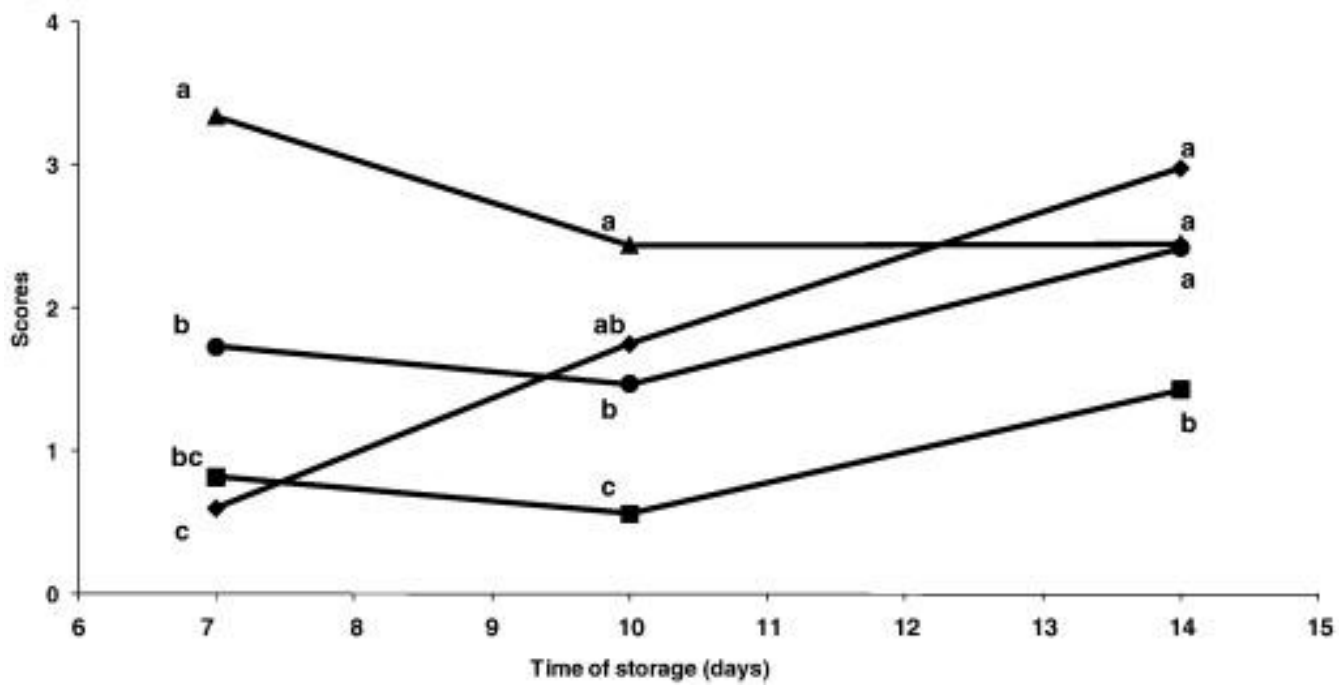


Figure 5
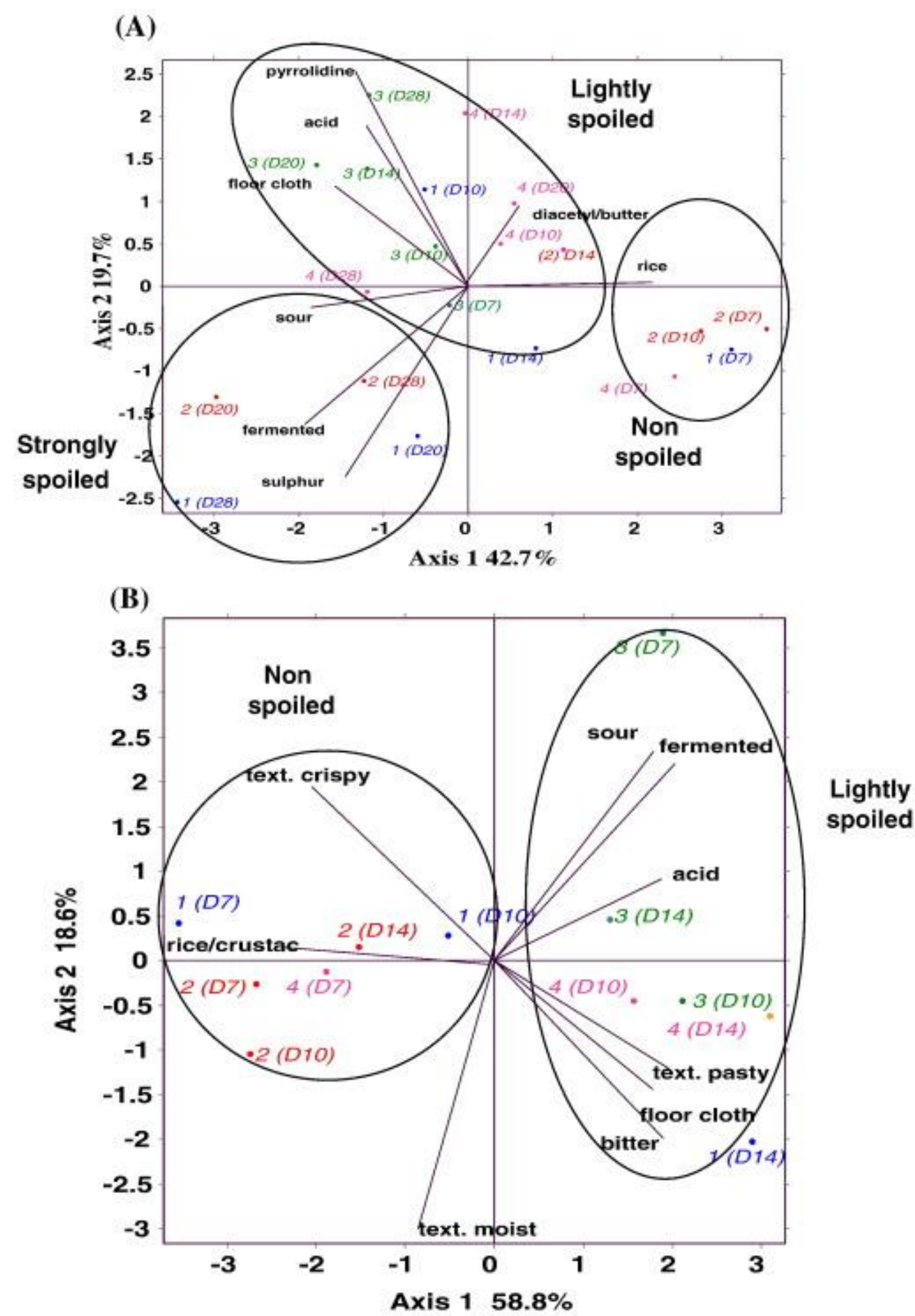
Figure 6

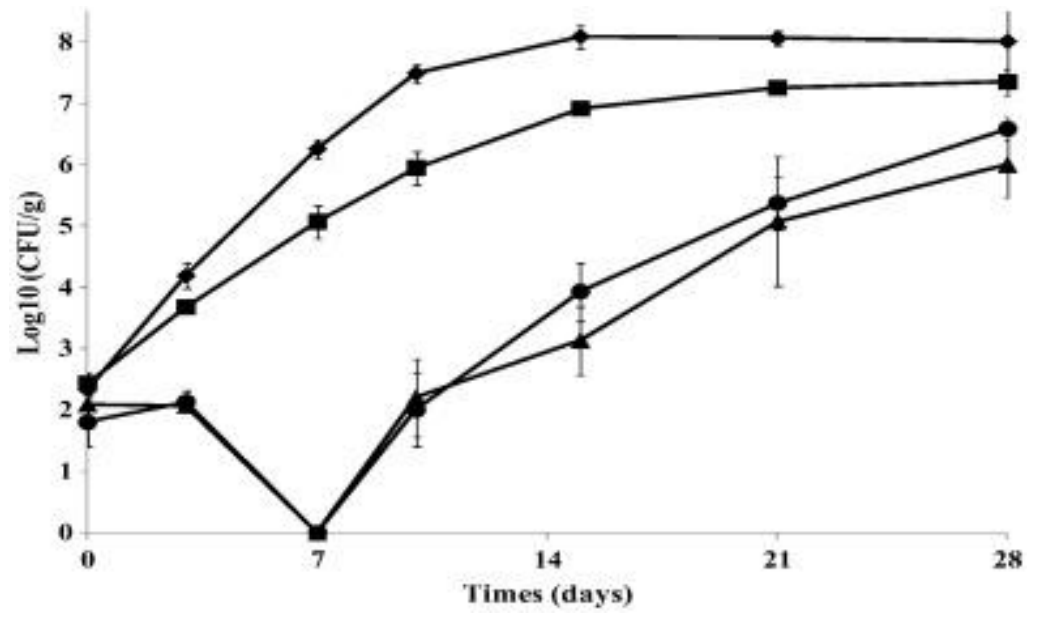

